\title{
Stem basic density and bark proportion of 45 woody species in young savanna coppice forests in Burkina Faso
}

\author{
Robert Nygård* and Björn Elfving \\ SLU, Department of Silviculture, 90183 Umeå, Sweden
}

(Received 24 June 1999; accepted 15 November 1999)

\begin{abstract}
In total 1287 sample trees were taken from 57 savanna woody species, representing 22 families in 5 stands, 5-14 years old, at 4 sites which has a mean annual precipitation of $620-785 \mathrm{~mm}$ in Burkina Faso. Stem discs were taken at one-meter intervals along the tree stem up to a diameter of $3 \mathrm{~cm}$. For 45 of these species, with more than 4 stems sampled, the stem basic density varied between 301-854 $\mathrm{kg} \mathrm{m}^{-3}$. Bark proportion of stem biomass varied between 9-53\%. Indications of decreased basic density and increased bark proportion with height of the stem and with decreased stem size was found for several species. Data presented provides a basis for the construction of models to convert standing woody volumes over bark to oven-dry mass whereby the bark proportion of the stem biomass can be determined.
\end{abstract}

specific gravity / humidity content / indigenous species / fuel-wood / biomass

Résumé - Densité basale de tronc et proportion d'écore de 45 espèces ligneuses issues de taillis dans une savane du Burkina Faso. Un échantillon de 1287 individus appartenant à 57 espèces et 22 familles de ligneux de savane a été coupé au Burkina Faso. Ces individus sont issus de 5 populations âgées de 5 à 14 ans provenant de 4 sites dont la pluviométrie est comprise entre 620 et $785 \mathrm{~mm}$. Des disques ont été pris à $1 \mathrm{~m}$ d'intervalle le long de la tige jusqu'à un diamètre de $3 \mathrm{~cm}$. Pour 45 de ces espèces comprenant plus de 4 tiges échantillonnées, la densité basale a varié entre 301 et $850 \mathrm{~kg} \mathrm{~m}^{-3}$ et la proportion d'écorce entre 9 et $53 \%$. Une diminution de la densité basale et une augmentation de la proportion d'écorce en fonction de la hauteur ont été observées pour plusieurs espèces. Les données présentées fournissent une base pour l'élaboration de modèles pour convertir les volumes de bois sur pied avec écorce en matière sèche d'étuve où la proportion d'écorce de la tige peut être déterminée.

gravité spécifique / taux d'humidité / espèces locales / bois de feu / biomasse

\section{ABBREVIATIONS}

$B D_{\mathrm{ub}} \quad$ Stem Basic Density under bark, $\mathrm{kg} \mathrm{m}^{-3}$

$B D_{\text {ub }}^{\text {height }}$ Disc Basic Density under bark per tree height, $\mathrm{kg} \mathrm{m}^{-3}$

$B D_{\text {ob }} \quad$ Stem Basic Density over bark, $\mathrm{kg} \mathrm{m}^{-3}$
$B_{M \%} \quad$ Stem Bark Mass Proportion on an oven-dry mass basis, \%

$B_{W \%}^{\text {height }} \quad$ Disc Bark Proportion on an oven-dry mass basis per tree height, \%

$B_{V \%} \quad$ Stem Bark Volume Proportion on a green volume basis, \%

* Correspondence and reprints

Tel. +46 9078658 72; Fax. 78676 69; e-mail: robert.nygard@ssko.slu.se 


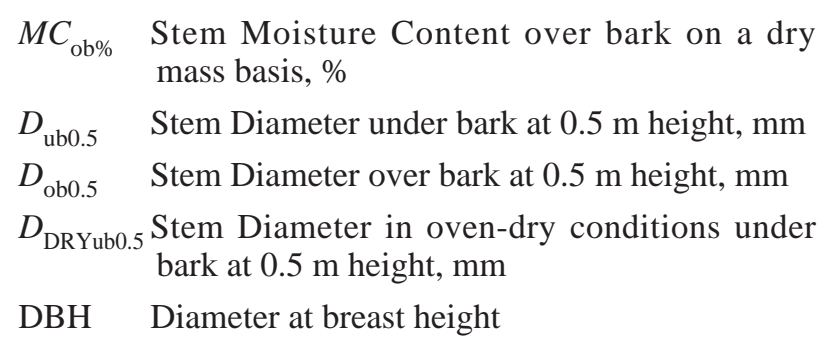

\section{INTRODUCTION}

In Sahel, fuel-wood has historically been collected from dead trees without bark, whereas today fuel-wood increasingly originates from the cutting of live woody stems [13], particularly in the vicinity of urban areas. In developing silvicultural systems for firewood production in the Sahel, short-rotation coppice silviculture $[7,10]$ or coppice with standards $[5,11]$ have been proposed. Rotation periods of at least 5 years and older depending on the woody species and required dimensions for harvesting has been suggested in savanna silviculture $[1,7]$. At present, a rotation of 20 years is tested in a large-scale operation at Burkina Faso for the supply of fuel-wood to the capital Ougadougou [5]. In fact, large forest areas in Sahel are now considered to have secondary coppice growth and their accompanying rotation periods are gradually getting shorter $[1,5]$.

Reliable estimates of the woody oven-dry biomass in coppice forests are needed for analyses of the fuel-wood balance in Sahel. Existing forest inventory data is reported in terms of standing woody volumes over bark but these volumes require basic density of a given species for the conversion to oven-dry mass $[8,10]$. However, species composition varies between different forests therefore conversion factors (volume to oven-dry mass) for a forest should be weighted by the frequency of occurrence of each species. At present, the conversion factor of 0.62 ton $\mathrm{m}^{-3}$ is used, independently of woody species and tree age, to calculate the woody biomass in Sahel [10]. Furthermore an assumed uniform bark volume proportion of $13 \%$, is used to calculate the available fuel-wood under bark.

Information on species basic density is a key factor for investigating calorific value and thus fuel-wood quality [1]. In general bark is inferior to wood in terms of basic density $[8,10]$. Another aspect of fuelwood quality is the unhealthy emission when bark is used for fuelwood. For instance high nitrogen concentrations in the bark of Acacia species have been reported to give high levels of nitrogen oxides when burning and therefore debarking is suggested [15]. Another argument for debarking is to reduce the nutrient removal from the for- est [16]. To analyse the consequences on fuelwood production of debarking there is a need to determine the difference in bark proportion between woody species.

In general, there is a variability of basic density among individuals of a given species, among geographical locations, with age and along stems [8, 17]. Since wood is a hygroscopic material both mass and volume varies with the moisture content, and volumes above the fibre saturation point are marginally affected, there are a variety of ways to calculate wood basic densities. The most appropriate measure for assessment of biomass is basic density, or oven-dry mass divided by wet volume [8]. The wet volume usually refers to wood samples soaked in water until saturation in the laboratory, which is relatively equivalent to green volume in standing trees $[6,8,12,17]$.

This study was performed in conjunction with a shortterm rotation management for fuel-wood production in natural savanna forests. The aim of this paper was to determine stem wood basic density and bark proportion for woody species in young coppice stands in Burkina Faso. This would provide tools for constructing models that convert green woody stem volume to oven-dry mass with and without bark per species $[5,8,10]$. The data is required in analysis of a regional or national fuel-wood balance to convert existing forest inventory data from woody volumes to oven-dry mass in young coppice stands. Further, data presented could also be used for discussions on the ecological implications of different fuel-wood management strategies.

\section{MATERIALS AND METHODS}

\subsection{Study sites}

The study was carried out in Burkina Faso, West Africa, in the tree- and shrub savanna zone [3] in the north Soudanian zone [9]. Mean annual precipitation and temperature, for the period 1983-1996, at the Ougadougou airport located close to the centre of the study area at $\left(12^{\circ} 25^{\prime} \mathrm{N}, 1^{\circ} 30^{\prime} \mathrm{E}\right)$ was $723 \mathrm{~mm}$ and $28^{\circ} \mathrm{C}$, respectively. The dry season lasts for 6 months according to the definition by Bagnouls and Gaussen [4]. Sample trees for determination of basic density were taken from 5 stands located at 4 sites (figure 1), all at an altitude of 300 m.a.s.l., and with an annual mean precipitation ranging between 620-785 mm (table I). Stands had emerged after clear-cut and varied in ages between 5 to 14 years when they were cut in 1996-97. Stand density varied between 635-1234 stem ha ${ }^{-1}$. One site, the Sa forest, is situated on a hydromorphic mineral of vertisoil type. The other three sites are located on leached grey ferruginous soils on sandy, sandy-clay or clayey-sand 
material. Many species sporadically occurred in a patchy spatial structure and it was suggested that vegetative regeneration from stumps, stools and roots dominated on a woody volume basis. Experimental sites of 4 ha were selected in representative areas of each forest and had been protected from fire since the last clear-cut in the early 1980's.

\subsection{Sampling procedure}

The experiment consisted of 16 adjacent square plots of $2500 \mathrm{~m}^{2}(50 \times 50 \mathrm{~m})$, grouped in 4 square blocks, one plot per block was randomly selected for clear-cutting and split in a grid of $25 \mathrm{~m}^{2}(5 \times 5 \mathrm{~m})$ plots [11]. Sample trees $>3 \mathrm{~cm} \mathrm{DBH}$ from different species and stands were selected in parity to their occurrence. Within species only one stem was sampled per $25 \mathrm{~m}^{2}$ plot or per stool and with even distribution of diameters. Sampling was carried out during the midst of the dry season, from February to May, and at this time few species had leaves. Every woody species encountered on each site was represented by at least one sample. Classification of woody plant stature in tree, bush and lianoid growth and identification of species and families follows Guinko [9].

\subsection{Mensuration of stem disc samples}

Cutting and weighing of tree disc samples were made less than one hour after felling the tree. Stem discs, $10 \mathrm{~cm}$ thick, were cut at every meter starting from $0.5 \mathrm{~m}$ up the height of the main stem until a diameter of $3 \mathrm{~cm}$ over bark was reached. If the sample position on the stem fell on a knot the cutting place was shifted up or down along the stem. Dead stems were not sampled. On discs taken at $0.5 \mathrm{~m}$ from the stump, diameter was measured by cross calipering over bark $\left(D_{\mathrm{ob} 0.5}\right)$ and under bark $\left(D_{\text {ub0.5 }}\right)$ in fresh condition and under bark $\left(D_{\text {DRYub0.5 }}\right)$ in oven-dry condition (see abbreviations).

Volume determination was made with a modified version of the water displacement method [12]. After placing 15 litres of water in a container, on an electronic balance $(1 \mathrm{~g})$ it was tarred. Immersion of a sample just under the water surface was done by hand with a needle, assumed to have negligible volume, attached to the sample. Dry mass was determined on an electronic balance $(1 \mathrm{~g})$ immediately after drying in an oven at $103 \pm 2{ }^{\circ} \mathrm{C}$ to constant mass, which took 4-5 days. Volume determination is made indoors on a saturated wood sample in Gonse, Tiogo and Yabo whereas in Sa forest volume determination was made with a portable electronic balance $(1 \mathrm{~g})$ on fresh disc samples in the forest. About half of all 1287 samples were taken in Sa forest and we

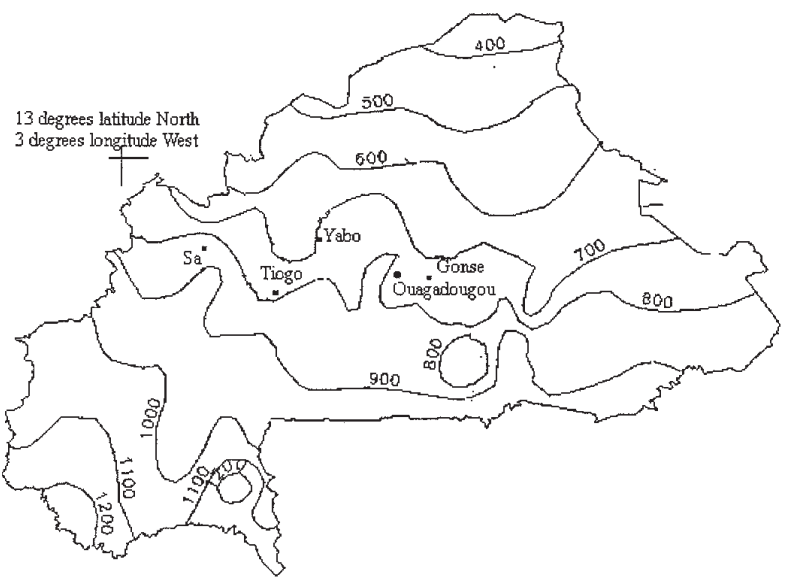

Figure 1. Rainfall patterns in mm per year and the geographical position of four forest stands: Sa, Tiogo, Yabo and Gonse and the capital of Burkina Faso, Ougadougou. Scale $1: 5.000 .000$.

assume these measurement systems gives equivalent result. Restoration of the green volume by saturation of the wood sample is an assumption in most studies determining wood basic density $[6,8,12,17]$.

\subsection{Calculations and statistical analysis}

For each stem basic density under bark $\left(B D_{\mathrm{ub}}\right)$ and over bark $\left(B D_{\mathrm{ob}}\right)$, bark mass percentage on a dry mass basis $\left(B_{M \%}\right)$, bark volume percentage on a green volume basis $\left(B_{V \%}\right)$ and moisture content over bark $\left(M C_{\mathrm{ob} \%}\right)$ were calculated by summing disc values taken from each main stem:

$\begin{aligned} B D_{\mathrm{ub}} & =\frac{\sum \text { ovendry disc massunder bark }}{\sum \text { freshdisc volumeunder bark }} \\ B D_{\mathrm{ob}} & =\frac{\sum \text { ovendry disc mass over bark }}{\sum \text { freshdisc volumeover bark }}\end{aligned}$

$B_{M \%}=\frac{\sum \text { ovendry disc mass over bark }-\sum \text { ovendry disc massunder bark }}{\sum \text { ovendry disc mass over bark }} * 100$

$\sum$ green disc volumeover bark $-\sum$ green disc volumeunder bark

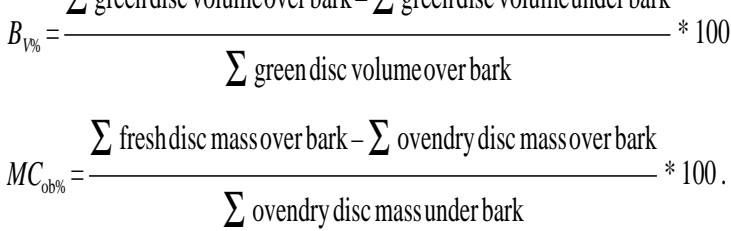


Table I. General data on investigated stands.

\begin{tabular}{|c|c|c|c|c|c|}
\hline & Gonse $10 *$ & Gonse $5 *$ & $\begin{array}{c}\text { Site } \\
\text { Sa } 14 *\end{array}$ & Tiogo $13 *$ & Yabo $13 *$ \\
\hline Species encountered & 28 & 34 & 24 & 44 & 19 \\
\hline Total number of sampled trees & 129 & 176 & 577 & 278 & 127 \\
\hline Stand density ( & 779 & 522 & 1234 & 777 & 655 \\
\hline Annual precipitation $\mathrm{mm}$ & 735 & 735 & 680 & 785 & 620 \\
\hline
\end{tabular}

DBH Diameter at Breast Height.

* The figure after the name of the site indicate stand age.

Analysis of covariance [18] was used to test the site effect per species by using $B D_{\mathrm{ub}}$ as a linear function of $D_{\text {ub } 0.5}$ for 11 ubiquitous species. After pooling samples from all stands mean values per species for the 45 species with more than 4 sample trees were calculated for diameter $\left(D_{\mathrm{ob} 0.5}, D_{\mathrm{ub0} 0.5}\right.$ and $\left.D_{\text {Dryub0.5 }}\right)$, basic density $\left(B D_{\mathrm{ub}}\right.$ and $\left.B D_{\mathrm{ob}}\right)$, bark percentage $\left(B_{M \%}\right.$ and $\left.B_{V \%}\right)$ and moisture content $\left(M C_{\mathrm{ob} \%}\right)$. Simple linear regressions were fit to $B D_{\mathrm{ub}}$ on $D_{\mathrm{ub} 0.5}$ and to $B_{M \%}$ on $D_{\mathrm{ub} 0.5}$ for 5 species. Mean $M C_{\mathrm{ob} \%}$ per species was fitted with a linear regression to the mean $B D_{\mathrm{ub}}$ per species for the 45 sampled species.

Mean values per stem height and their standard errors were calculated for basic density under bark ( $\left.B D_{\text {ub }}^{\text {height }}\right)$ and bark percentage $\left(B_{W \%}^{\text {height }}\right)$. For Anogeissus leiocarpus and Acacia seyal Restricted Maximum Likelihood (REML) was used for estimation of the variance component of $B_{\mathrm{ub}}^{\text {height }}$ among trees with the following model;

$B D_{\mathrm{ub}}^{\text {height }}{ }_{i j}=\beta_{0}+\beta_{1} * D_{\mathrm{ub} 0.5 i}+\beta_{2} *$ height $_{i j}+\alpha_{i}+\varepsilon_{i j}$

where $\beta_{0}, \beta_{1}$ and $\beta_{2}$ are coefficients, $\alpha$ is a random tree effect and $i$ is the tree number and $j$ is the disc number within the tree. All $\alpha_{i}$ and $\varepsilon_{i j}$ are assumed to be independent and have a normal distribution with mean zero. Discs were numbered starting from $i=1$ at the $0.5 \mathrm{~m}$ level. The significance level of all statistical tests was 0.05 and the word "mean" was applied for arithmetic mean. Statistical analysis was performed with SPSS 8.0.0 and SAS 6.12.

\section{RESULTS}

The number of species encountered per stand, varied from 19 to 44 (table I), and few species were present on all sites. Out of totally 57 species representing 22 families, 34 had a tree stature, 16 were bushes and 7 had a lianoid growth (table $I I$ ). Species mean $D_{\text {ob0.5 }}$ ranged from $20 \mathrm{~mm}$ to $95 \mathrm{~mm}$ indicating a large difference in growth after clear-cutting. No significant site effect on species basic density was found among the 11 ubiquitous species tested (table II). For the 45 species with more than 4 sample trees the range of $B D_{\mathrm{ub}}$ was $301-854 \mathrm{~kg}$ $\mathrm{m}^{-3}$ (table $\left.I I I\right)$. Several species had similar $B D_{\mathrm{ub}}$ and within species variation was often larger than the variation between species mean $B D_{\mathrm{ub}}$. The range of $B D_{\mathrm{ob}}$ was 253-807 kg m $\mathrm{kg}^{-3}$ and double bark in percentage of $D_{\mathrm{ob} 0.5}$ ranged from $9 \%$ to $37 \%$. Wood shrinkage expressed in terms of percentage contraction of $D_{\mathrm{ub} 0.5}$ ranged from $2 \%$ to $10 \%$. The $B_{M \%}$ ranged from $9 \%$ to $53 \%$ and $B_{V \%}$ ranged from $11 \%$ to $51 \%$. $M C_{\mathrm{ob} \%}$ ranged from $34 \%$ to $294 \%$.

In general fast-growing species like Bombax costatum with a $D_{\mathrm{ob} 0.5}$ of $85 \mathrm{~mm}$ had low $B D_{\mathrm{ob}}\left(253 \mathrm{~kg} \mathrm{~m}^{-3}\right)$ and slow-growing species like Dicrostachys cinerea with a $D_{\text {ob0.5 }}$ of $46 \mathrm{~mm}$ had high $B D_{\mathrm{ob}}\left(787 \mathrm{~kg} \mathrm{~m}^{-3}\right)$. Furthermore fast-growing species had large bark thickness $\left(D_{\mathrm{ob} 0.5}-D_{\mathrm{ub} 0.5}\right)$ for instance Bombax costatum had $32 \mathrm{~mm}$, or $37 \%$ expressed as a percentage of $D_{\mathrm{ob} 0.5}$ and the opposite was found for species with low $D_{\text {ob } 0.5}$ like Dicrostachys cinerea, which had $7 \mathrm{~mm}$ or $17 \%$. BD $\mathrm{ub}$ was less than $B D_{\mathrm{ob}}$ for fast-growing species like Lannea sp., Commiphora africana, Detarium microcarpum and Entada africana indicating a higher basic density for bark than for wood. The difference found between species double bark thickness at $0.5 \mathrm{~m}$ stem height was also found in the difference between species $B_{M \%}$ and $B_{V \%}$ for the whole stem. There was no pattern found in the wood shrinkage between species with regard to $B D_{\mathrm{ub}}$. Coefficient of determination for species mean $M C_{\mathrm{ob} \%}$ for 45 species on species mean $B D_{\text {ob }}$ was $83 \%$ with intercept $=350.9$ and slope $=-0.417$.

For Anogeissus leiocarpus and Acacia seyal, representing two species with a tree stature, the variance in disc basic density ( $\left.B D_{\mathrm{ub}}^{\text {height }}\right)$ between trees was larger than the variance within trees, (model 6) $56 \%$ and $62 \%$, respectively (table $V$ ). Estimates of coefficients $\beta_{1}$ and $\beta_{2}$, showed that disc basic density ( $\left.B D_{\mathrm{ub}}^{\text {height }}\right)$ augmented 
Table II. Stem basic density $\left(\mathrm{kg} \mathrm{m}^{-3}\right)$ under bark for 57 savanna woody species on 4 sites in Burkina Faso.

\begin{tabular}{|c|c|c|c|c|c|c|c|}
\hline Species & Family & Stature & Gonse10 & Gonse 5 & $\begin{array}{l}\text { site } \\
\text { Sa14 }\end{array}$ & Tiogo13 & Yabo13 \\
\hline Acacia ataxacantha DC. & Mimosaceae & $\mathrm{L}$ & & & 694 & & \\
\hline Acacia dudgeoni Craib ex. Holl. & Mimosaceae & $\mathrm{T}$ & 723 & 768 & & 671 & 701 \\
\hline Acacia gourmaensis A. Chev.* & Mimosaceae & $\mathrm{T}$ & 772 & 713 & & & \\
\hline Acacia macrostachya Reichenb. ex Benth. & Mimosaceae & B & 700 & 736 & & 761 & 763 \\
\hline Acacia pennata (Linn.) Willd. & Mimosaceae & $\mathrm{L}$ & 710 & & 836 & 712 & 705 \\
\hline Acacia senegal (Linn.) Willd. & Mimosaceae & $\mathrm{T}$ & & 767 & & & 683 \\
\hline Acacia seyal Del. & Mimosaceae & $\mathrm{T}$ & 728 & 734 & 749 & 711 & \\
\hline Albizzia chevalieri Harms & Mimosaceae & $\mathrm{T}$ & & & 642 & & \\
\hline Anogeissus leiocarpus (DC.) Guill. et Perr.* & Combretaceae & $\mathrm{T}$ & 753 & 708 & 709 & 750 & 785 \\
\hline Balanites aegyptiaca (L.) Del. & Balanitaceae & $\mathrm{T}$ & 668 & 636 & 702 & 659 & 695 \\
\hline Bombax costatum Pellegr. et Vuillet & Bombacaceae & $\mathrm{T}$ & 311 & 286 & 305 & & \\
\hline Boscia senegalensis (Pers.) Lam. ex Poir. & Capparacea & $\mathrm{B}$ & & & 700 & & \\
\hline Boswellia dalzielli Hutch. & Burceraceae & $\mathrm{T}$ & & & 720 & & \\
\hline Butyrospermum paroxum $* *$ & Sapotaceae & $\mathrm{T}$ & 675 & 686 & & 712 & \\
\hline Capparis sepiaria & Caparacea & $\mathrm{L}$ & & & 627 & & \\
\hline Cassia sieberiana DC. & Caesalpiniaceae & B & 714 & 744 & & 700 & \\
\hline Cassia singueana Del. & Caesalpiniaceae & B & & 690 & & & \\
\hline Combretum aculeatum Vent. & Combretaceae & $\mathrm{L}$ & & 687 & & & 683 \\
\hline Combretum fragrans $\mathrm{F}$. Hoffm. & Combretaceae & $\mathrm{T}$ & & & & 635 & \\
\hline Combretum glutinosum Perr. ex DC.* & Combretaceae & $\mathrm{T}$ & 694 & 660 & & 700 & 720 \\
\hline Combretum micranthum $\mathrm{G}$. Don * & Combretaceae & B & & 768 & 707 & 766 & 798 \\
\hline Combretum nigricans Lepr. ex Guill. et Perr. * & Combretaceae & $\mathrm{T}$ & & & 758 & 761 & 746 \\
\hline Commiphora africana (A. Rich.) Engl.* & Burceraceae & $\mathrm{T}$ & 332 & 402 & 367 & 328 & 347 \\
\hline Crossopteryx febrifuga (Afzel. ex G. Don) Benth. & Rubiaceae & $\mathrm{T}$ & 631 & 620 & & 602 & \\
\hline Dalbergia melanoxylon Guill. et Perr. & Papilionaceae & $\mathrm{T}$ & & & 817 & & 804 \\
\hline Detarium microcarpum Guill. et Perr. & Caesalpiniaceae & $\mathrm{T}$ & & 515 & & 582 & \\
\hline Dicrostachys cinerea (L.) Wight et Arn.* & Mimosaceae & $\mathrm{T}$ & 871 & 831 & 844 & 893 & 866 \\
\hline Diospyros mespiliformis Hoschst. ex A.DC. & Ebenaceae & $\mathrm{T}$ & & & & 642 & \\
\hline Entada africana Guill. et Perr. & Mimosaceae & $\mathrm{B}$ & 513 & 496 & & 558 & \\
\hline Feretia apodanthera Del. & Rubiaceae & B & 686 & 676 & 647 & 695 & \\
\hline Gardenia ternifolia Schum. et Thonn. & Rubiaceae & B & & & & 655 & \\
\hline Grewia bicolor Juss.* & Tiliaceae & $\mathrm{T}$ & 764 & 789 & 740 & 799 & 799 \\
\hline Grewia flavescens Juss. & Tiliaceae & $\mathrm{L}$ & & & 654 & 720 & \\
\hline Grewia mollis Juss. & Tiliaceae & $\mathrm{B}$ & & & 716 & 714 & \\
\hline Guiera senegalensis J. F. Gmel. in L. & Combretaceae & B & 656 & 636 & 609 & 692 & 695 \\
\hline Lannea acida A. Rich.* & Anacardiaceae & $\mathrm{T}$ & 463 & 463 & 463 & 471 & 462 \\
\hline Lannea microcarpa Engl. et Krause & Anacardiaceae & $\mathrm{T}$ & & & & 464 & 464 \\
\hline Mitragyna inermis (Willd.) O. Kotze. & Rubiaceae & $\mathrm{T}$ & & & 600 & 563 & \\
\hline Piliostigma reticulatum (DC.) & Caesalpiniaceae & B & 671 & 642 & & 628 & \\
\hline Piliostigma thonningii (Schum.) Miln-Red. & Caesalpiniaceae & B & & 705 & & 655 & \\
\hline Prosopis africana Taub. & Mimosaceae & $\mathrm{T}$ & & & & 687 & \\
\hline Pterocarpus lucens Lepr. ex Guill. et Perr.* & Papilionaceae & $\mathrm{T}$ & & & 805 & & 866 \\
\hline Pterocarpus erinaceus Poir. & Papilionaceae & $\mathrm{T}$ & 657 & 620 & & 672 & \\
\hline Saba senegalensis (A. DC.) Pichon & Apocynaceae & $\mathrm{L}$ & & & & 523 & \\
\hline Sclerocarya birrea (A. Rich.) Hoschst.* & Anacardiaceae & $\mathrm{T}$ & 503 & 461 & 496 & 535 & 550 \\
\hline Securinega virosa (Roxb. Ex Willd.) Baill. & Euphorbiaceae & $\mathrm{B}$ & & 680 & & 688 & \\
\hline Sterculia setigera Del. & Sterculiaceae & $\mathrm{T}$ & 322 & & & 292 & \\
\hline Stereospermum kunthianum Cham. & Bignoniaceae & $\mathrm{T}$ & 595 & 622 & & 693 & \\
\hline Strychnos spinosa Lam. & Loganiaceae & $\mathrm{B}$ & & & & 693 & \\
\hline Tamarindus indica $\mathrm{L}$. & Caesalpiniaceae & $\mathrm{T}$ & 772 & 750 & & 769 & \\
\hline Terminalia avicennioides Guill. et Perr. & Combretaceae & $\mathrm{T}$ & 648 & 631 & & 636 & \\
\hline Terminalia laxiflora Engl. & Combretaceae & $\mathrm{T}$ & & 655 & & 663 & \\
\hline $\begin{array}{l}\text { Terminalia macroptera Guill. et Perr. } \\
\text { Xeroderris stuhlmannii (Taub.) }\end{array}$ & Combretaceae & $\mathrm{T}$ & & 590 & & 622 & \\
\hline Mendonca et E. P. Sousa & Papilionaceae & $\mathrm{T}$ & & & & 565 & \\
\hline Ximenia americana $\mathrm{L}$. & Olacacea & B & 623 & 644 & 651 & 654 & \\
\hline Ziziphus mauritiana Lam. & Rhamnacea & B & 517 & & & & \\
\hline Ziziphus mucronata Willd. & Rhamnacea & B & & & & 645 & \\
\hline
\end{tabular}

* Tested for stand effect.

** Synonomous Vittelaria paradoxa C.F. Gaertn.

T: Tree.

B: Bush.

L: Lianoid growth. 
Table III. Mean dendrological parameters for 45 savanna woody species in the age 5-14 years in Burkina Faso.

\begin{tabular}{|c|c|c|c|c|c|c|c|c|c|}
\hline Species & $N$ & $\begin{array}{c}B D_{\mathrm{ub}^{-3}} \\
\mathrm{~kg} \mathrm{~m}^{-3}\end{array}$ & $\begin{array}{c}B D_{\mathrm{ob}} \\
\mathrm{kg} \mathrm{m}^{-3}\end{array}$ & $\underset{\mathrm{ob0} 0.5}{D_{\mathrm{m}}}$ & $B_{\text {thick }}$ & shrinkage & $\begin{array}{c}B_{M \%} \\
\text { percentage }\end{array}$ & $B_{V \%}$ & $M C_{\mathrm{ob}}$ \\
\hline Acacia ataxacantha & 29 & $694(33)$ & 707 & 39 & 14 & 4 & $18(7)$ & 18 & 34 \\
\hline Acacia dudgeoni & 6 & $728(33)$ & 701 & 51 & 25 & 5 & $33(5)$ & 35 & 78 \\
\hline Acacia gourmaensis & 27 & $748(57)$ & 624 & 57 & 29 & 4 & $31(8)$ & 42 & 72 \\
\hline Acacia macrostachya & 33 & $759(40)$ & 727 & 60 & 21 & 4 & $28(4)$ & 31 & 60 \\
\hline Acacia pennata & 11 & $744(75)$ & 728 & 39 & 13 & 3 & $13(4)$ & 14 & 50 \\
\hline Acacia senegal & 6 & $738(67)$ & 671 & 46 & 25 & 2 & $31(6)$ & 37 & 86 \\
\hline Acacia seyal & 85 & $751(37)$ & 702 & 67 & 17 & 3 & $24(5)$ & 29 & 68 \\
\hline Albizzia chevalieri & 8 & $642(46)$ & 574 & 47 & 23 & 3 & $26(9)$ & 34 & 80 \\
\hline Anogeissus leiocarpus & 151 & $720(45)$ & 721 & 65 & 14 & 4 & $21(5)$ & 21 & 53 \\
\hline Balanites aegyptiaca & 17 & $677(50)$ & 651 & 80 & 18 & 3 & $33(9)$ & 35 & 67 \\
\hline Bombax costatum & 19 & $306(35)$ & 253 & 87 & 37 & 6 & $41(7)$ & 51 & 237 \\
\hline Boscia senegalensis & 12 & $700(56)$ & 682 & $* *$ & $* *$ & $* *$ & $31(4)$ & 33 & 62 \\
\hline Boswellia dalzielli & 16 & $719(51)$ & 730 & 20 & 23 & 7 & $35(6)$ & 34 & 52 \\
\hline Butyrospermum paradoxum & 20 & $696(56)$ & 639 & 72 & 22 & 4 & $32(5)$ & 37 & 89 \\
\hline Capparis sepiaria & 12 & $636(54)$ & 683 & $* *$ & $* *$ & $* *$ & $22(8)$ & 17 & 83 \\
\hline Cassia siberiana & 5 & $720(25)$ & 721 & 69 & 10 & 2 & $17(1)$ & 17 & 59 \\
\hline Combretum fragrans & 5 & $635(21)$ & 642 & 49 & 19 & 2 & $26(1)$ & 25 & 84 \\
\hline Combretum glutinosum & 49 & $686(41)$ & 674 & 56 & 14 & 3 & $21(6)$ & 23 & 74 \\
\hline Combretum mircathum & 78 & $736(54)$ & 730 & 43 & 11 & 3 & $15(3)$ & 16 & 41 \\
\hline Combretum nigricans & 43 & $751(34)$ & 751 & 62 & 9 & 2 & $17(4)$ & 17 & 55 \\
\hline Commiphora africana & 80 & $365(29)$ & 381 & 56 & 19 & 3 & $33(6)$ & 30 & 164 \\
\hline Crossopteryx febrifuga & 10 & $610(16)$ & 623 & 63 & 16 & 4 & $27(6)$ & 26 & 81 \\
\hline Dalbergia melanoxylon & 36 & $819(25)$ & 794 & 48 & 15 & 5 & $18(3)$ & 21 & 48 \\
\hline Detarium microcarpum & 8 & $565(38)$ & 614 & 75 & 25 & 4 & $44(12)$ & 39 & 95 \\
\hline Dicrostachys cinerea & 72 & $854(35)$ & 787 & 46 & 17 & 5 & $18(4)$ & 25 & 39 \\
\hline Entada africana & 16 & $517(46)$ & 537 & 64 & 23 & 3 & $37(8)$ & 35 & 128 \\
\hline Feretia apodanthera & 40 & $671(33)$ & 661 & 34 & 15 & 5 & $21(4)$ & 23 & 57 \\
\hline Grewia bicolor & 45 & $780(46)$ & 761 & 52 & 20 & 7 & $24(4)$ & 26 & 40 \\
\hline Grewia flavescens & 12 & $671(106)$ & 621 & 21 & 18 & 10 & $22(11)$ & 29 & 44 \\
\hline Grewia mollis & 13 & $715(31)$ & 719 & 48 & 22 & 6 & $29(4)$ & 29 & 43 \\
\hline Guiera senegalensis & 20 & $681(34)$ & 669 & 55 & 10 & 6 & $9(2)$ & 11 & 46 \\
\hline Lannea acida & 31 & $465(25)$ & 545 & 75 & 30 & 4 & $53(9)$ & 46 & 141 \\
\hline Lannea mirocarpa & 6 & $468(10)$ & 510 & 73 & 27 & 4 & $40(3)$ & 35 & 153 \\
\hline Piliostigma reticulatum & 18 & $641(48)$ & 612 & 57 & 23 & 3 & $29(8)$ & 32 & 97 \\
\hline Piliostigma thonningii & 6 & $664(37)$ & 616 & 60 & 27 & 3 & $35(6)$ & 40 & 95 \\
\hline Prosopis africana & 6 & 687 (13) & 650 & 93 & 15 & 4 & $25(3)$ & 29 & 83 \\
\hline Pterocarpus lucens & 42 & $830(45)$ & 807 & 59 & 11 & 4 & $15(3)$ & 18 & 37 \\
\hline Pterocarpus erinaceus & 11 & $656(41)$ & 623 & 61 & 17 & 2 & $36(10)$ & 39 & 88 \\
\hline Sclerocarya birrea & 49 & $509(45)$ & 500 & 77 & 20 & 3 & $30(4)$ & 31 & 145 \\
\hline Securinega virosa & 7 & $684(33)$ & 673 & 40 & 9 & 5 & $12(3)$ & 14 & 55 \\
\hline Sterculia setigera & 7 & $301(58)$ & 347 & 95 & 21 & 5 & $36(7)$ & 23 & 294 \\
\hline Strychnos spinosa & 6 & $693(31)$ & 629 & 60 & 12 & 2 & $20(3)$ & 27 & 73 \\
\hline Tamarindus indica & 12 & $767(25)$ & 699 & 52 & 20 & 4 & $24(5)$ & 30 & 50 \\
\hline Terminalia avicennoides & 6 & $638(18)$ & 617 & 61 & 24 & 4 & $39(6)$ & 41 & 73 \\
\hline Ximenia americana & 12 & $646(22)$ & 614 & 53 & 26 & 5 & $38(7)$ & 41 & 67 \\
\hline
\end{tabular}

$N \quad$ Number of stems sampled.

$B D_{\text {ub }}$ (st. dev.) Stem Basic Density under bark in $\mathrm{kg} \mathrm{m}^{-3}$, standard deviation in brackets.

$B D_{\mathrm{ob}} \quad$ Stem Basic Density over bark in $\mathrm{kg} \mathrm{m}^{-3}$.

$D_{\text {ob0.5 }} \quad$ Stem Diameter over bark in $\mathrm{mm}$ at 0.5 meter height.

$B_{\text {thick }} \quad$ Double bark thickness $\left(D_{\mathrm{ob} 0.5}-D_{\mathrm{ub} 0.5}\right)$ expressed as a percetage of $D_{\mathrm{ob} 0.5}$

Shrinkage Radial wood shrinkage $\left(D_{\mathrm{ub} 0.5}-D_{\text {DRYub0.5 }}\right)$ expressed as percentage of $D_{\text {ub0.5 }}$.

$B_{M \%}$ (st. dev.) Stem Bark Weight Proportion (\%) on a dry weight basis, standard deviation in brackets.

$B_{V \%} \quad$ Stem Bark Volume Proportion (\%) on a green volume basis.

$M C_{\mathrm{ob}} \quad$ Stem Moisture Content over bark $(\%)$ on a dry weight basis.

** $\quad$ Missing values. 
Table IV. Stem basic density under bark $\left(B D_{\mathrm{ub}}\right)$ and stem bark proportion $\left(B_{M \%}\right)$ as function of tree size $\left(D_{\mathrm{ub} 0.5}\right)$ for 5 savanna woody species.

\begin{tabular}{lccc}
\hline Species & Parameter estimates & $r^{2}$ & $p$-value \\
\hline Anogeissus leiocarpus & $B D_{\mathrm{ub}}=663+1.0 * D_{\mathrm{ub} 0.5}$ & 16 & 0,000 \\
Anogeissus leiocarpus & $B_{M \%}=0.28-0.0011 * D_{\mathrm{ub} 0.5}$ & 21 & 0,000 \\
Acacia seyal & $B D_{\mathrm{ub}}=705+0.7 * D_{\mathrm{ub} 0.5}$ & 6 & 0,018 \\
Acacia seyal & $B_{M \%}=0.36-0.0021 * D_{\mathrm{ub} 0.5}$ & 54 & 0,000 \\
Combrethum glutinosum & $B D_{\mathrm{ub}}=637+1.0 * D_{\mathrm{ub} 0.5}$ & 17 & 0,003 \\
Combrethum glutinosum & $B_{M \%}=0.31-0.0019 * D_{\mathrm{ub} 0.5}$ & 28 & 0,000 \\
Combrethum micranthum & $B D_{\mathrm{ub}}=610+3.2 * D_{\mathrm{ub} 0.5}$ & 24 & 0,000 \\
Combrethum micranthum & $B_{M \%}=0.22-0.0018 * D_{\mathrm{ub} 0.5}$ & 0,058 \\
Dicrostachys cinerea & $B D_{\mathrm{ub}}=820+0.7 * D_{\mathrm{ub} 0.5}$ & 23 & 0,000 \\
Dicrostachys cinerea & $B_{M \%}=0.25-0.0017 * D_{\mathrm{ub} 0.5}$ & 32 &
\end{tabular}

Table V. Variation in disc basic density within and between trees.

\begin{tabular}{|c|c|c|c|c|c|c|c|c|}
\hline & \multicolumn{2}{|c|}{ Anogeissus leiocarpus } & \multicolumn{6}{|c|}{ Acacia seyal } \\
\hline & Variance & $\%$ & & & Variance & $\%$ & & \\
\hline between trees & 1350 & 56 & & & 2226 & 62 & & \\
\hline within trees and error & 1070 & 44 & & & 1346 & 38 & & \\
\hline variation with fixed effects & 2420 & 100 & & & 3572 & 100 & & \\
\hline variation without fixed effects & 3089 & & & & 3776 & & & \\
\hline Fixed effects & Coefficient & SE & $\mathrm{DF}$ & $p$-values & Coefficient & SE & $\mathrm{DF}$ & $p$-values \\
\hline intercept & 696,72 & 12,12 & 133 & 0,0001 & 734,76 & 14,74 & 113 & 0,0001 \\
\hline tree size $\left(D_{0.5}\right)$ & 0,81 & 0,21 & 328 & 0,0001 & 0,48 & 0,25 & 288 & 0,0616 \\
\hline height level $(i=0.5-6.5)$ & $-23,51$ & 1,48 & 328 & 0,0001 & $-10,21$ & 1,58 & 288 & 0,0001 \\
\hline
\end{tabular}

with tree size $\left(D_{\mathrm{ub} 0.5}\right)$ and declined with height along the stem (table $V$ ). No interaction effect between tree size $\left(D_{\mathrm{ub} 0.5}\right)$ and height was found. Significant differences in mean $B D_{\text {ub }}^{\text {height }}$ with height along the stem between the first two or three meters up the stem were also found for several species in table VI. The $r^{2}$ for fitting $B D_{\mathrm{ub}}$ on $D_{\text {ub0.5 }}$ was low and ranged from 5-28\% for the 5 species tested (table $I V$ ), however the tendency was clear with increasing $B D_{\mathrm{ub}}$ with increased tree size. Corresponding $r^{2}$ for $B_{M \%}$ was also low and ranged from $24-54 \%$ but with decreasing $B_{M \%}$ with increased tree size.

\section{DISCUSSION}

During the 1980's, the Ministry of Forestry in Burkina Faso established plots on several sites that were representative forests in the country to analyse the production in short-term rotations with clear-cutting methods. The four sites in this study were selected to cover the range of site conditions in the north Soudanian zone. Yabo is the most arid site, situated at the border to the bush steppe in the south Sahel zone while Tiogo is the least arid close to the south Soudanian zone (table I, figure 1). Sa is bordering the tree savanna and situated on a vertisol with a stand density about twice as high compared the other stands. Given the difference in site conditions we wanted to check for variation in $B D_{\mathrm{ub}}$ between sites within species before pooling samples from all sites, but no stand effects on $B D_{\mathrm{ub}}$ were found. The test was made for 11 more ubiquitous species, sufficiently represented in more than one stand.

If studies would be made to closer examine site effects on species $B D_{\mathrm{ub}}$, very large samples are needed, since the variation between trees is large as indicated in this study e.g. Anogeissus leiocarpus and Acacia seyal. These two species were selected, for the analyses of variance components (model 6), because they were frequently sampled and had long stems providing several samples per tree. The parameter estimate for stem height $\left(\beta_{2}\right.$ in model 6) was $-23.51 \mathrm{~kg} \mathrm{~m}^{-3} \mathrm{~m}^{-1}$ for Anogeissus leiocarpus (table V). In the case of Anogeissus leiocarpus this represents about a $10 \%$ decrease in $B D_{u b}$ on four meters and this was also evident in table VI. However, 
Table VI. Basic density $\left(\mathrm{kg} \mathrm{m}^{-3}\right)$ under bark per tree height in meter starting at stump for a savanna coppice forest in the age 5-14 years in Burkina Faso.

\begin{tabular}{|c|c|c|c|c|c|c|c|c|c|c|c|c|c|c|}
\hline \multirow[b]{3}{*}{ species } & \multicolumn{14}{|c|}{ tree height in meter } \\
\hline & \multicolumn{2}{|c|}{0,5} & \multicolumn{2}{|c|}{1,5} & \multicolumn{2}{|c|}{2,5} & \multicolumn{2}{|c|}{3,5} & \multicolumn{2}{|c|}{4,5} & \multicolumn{2}{|c|}{5,5} & \multicolumn{2}{|c|}{6,5} \\
\hline & M & SE & M & SE & M & SE & M & SE & M & SE & M & SE & M & SE \\
\hline Acacia ataxacantha & 732 & 8 & 699 & 12 & 673 & 14 & 625 & 33 & 633 & 33 & . & . & . & \\
\hline Acacia dudgeoni & 725 & 14 & 701 & 17 & & & & . & & . & r & . & . & . \\
\hline Acacia gourmaensis & 746 & 15 & 731 & 16 & 708 & 31 & 667 & & 676 & . & . & . & 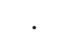 & . \\
\hline Acacia macrostachya & 782 & 8 & 726 & 8 & 703 & 11 & 664 & 22 & 659 & . & . & . & . & . \\
\hline Acacia pennata & 756 & 22 & 694 & 19 & 660 & & . & . & . & . & . & . & . & . \\
\hline Acacia senegal & 750 & 23 & 723 & 37 & 792 & 37 & & & & & & & & \\
\hline Acacia seyal & 753 & 5 & 741 & 5 & 737 & 7 & 733 & 11 & 705 & 25 & 718 & 37 & 690 & 30 \\
\hline Albizzia chevalieri & 649 & 20 & 639 & 13 & 600 & 0 & 571 & & & & & & . & . \\
\hline Anogeissus leiocarpus & 738 & 4 & 704 & 4 & 684 & 5 & 667 & 10 & 686 & 18 & 704 & 4 & . & . \\
\hline Balanites aegyptiaca & 682 & 12 & 671 & 15 & 687 & 17 & 659 & & 623 & . & . & . & . & . \\
\hline Bombax costatum & 319 & 8 & 295 & 10 & 281 & 9 & 281 & 7 & . & . & . & . & . & . \\
\hline Boscia senegalensis & 675 & 29 & . & . & . & . & . & . & . & . & . & . & . & . \\
\hline Boswellia dalzielli & 720 & 13 & & & & & & & & & . & . & . & . \\
\hline Butyrospermum paradoxum & 722 & 12 & 681 & 14 & 692 & 18 & 663 & 13 & 640 & 32 & . & . & . & . \\
\hline Capparis sepiaria & 627 & 25 & & & & & & . & . & . & . & . & . & . \\
\hline Cassia siberiana & 744 & 11 & 700 & 17 & 699 & 16 & 625 & . & . & . & . & . & 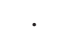 & . \\
\hline Combretum fragrans & 671 & 32 & 643 & 13 & 579 & 40 & & & . & . & . & . & . & . \\
\hline Combretum glutinosum & 697 & 6 & 674 & 7 & 675 & 11 & 629 & 16 & . & . & . & . & . & . \\
\hline Combretum mircathum & 746 & 6 & 722 & 6 & 701 & 10 & 740 & 19 & . & . & . & . & . & . \\
\hline Combretum nigricans & 776 & 6 & 725 & 6 & 698 & 10 & 688 & 15 & . & . & . & . & . & . \\
\hline Commiphora africana & 359 & 4 & 364 & 4 & 382 & 5 & 402 & 17 & . & . & . & . & . & . \\
\hline Crossopteryx febrifuga & 624 & 9 & 589 & 9 & 584 & 11 & 556 & & & & . & . & 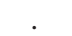 & . \\
\hline Dalbergia melanoxylon & 826 & 7 & 798 & 12 & 799 & 9 & 809 & 17 & 847 & 28 & . & . & 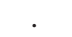 & . \\
\hline Detarium microcarpum & 582 & 15 & 551 & 16 & 533 & 16 & 531 & 13 & . & . & . & . & . & . \\
\hline Dicrostachys cinerea & 866 & 6 & 831 & 6 & 806 & 14 & 810 & & 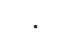 & . & . & . & . & . \\
\hline Entada africana & 518 & 13 & 521 & 12 & 551 & 15 & 563 & 40 & 508 & . & . & . & . & . \\
\hline Feretia apodanthera & 666 & 8 & 659 & 9 & 625 & 29 & 672 & & 691 & . & . & . & 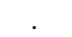 & . \\
\hline Grewia bicolor & 788 & 10 & 752 & 8 & 725 & 20 & 697 & 52 & . & . & . & . & . & . \\
\hline Grewia flavescens & 677 & 32 & 655 & 29 & 600 & & & . & . & . & . & . & . & . \\
\hline Grewia mollis & 729 & 12 & 703 & 15 & 669 & 23 & 733 & . & . & . & . & . & . & . \\
\hline Guiera senegalensis & 697 & 8 & 668 & 8 & 635 & 7 & 629 & & . & & . & . & . & . \\
\hline Lannea acida & 456 & 6 & 464 & 8 & 452 & 14 & 462 & 13 & 417 & 83 & . & . & 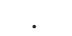 & . \\
\hline Lannea mirocarpa & 475 & 4 & 461 & 8 & 441 & 11 & 444 & & . & . & . & . & . & . \\
\hline Piliostigma reticulatum & 655 & 11 & 628 & 10 & 590 & 17 & 580 & 11 & . & . & . & . & . & . \\
\hline Piliostigma thonningii & 680 & 16 & 637 & 19 & 642 & & & & & & . & . & 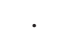 & . \\
\hline Prosopis africana & 716 & 9 & 662 & 11 & 636 & 8 & 632 & 3 & 673 & 31 & & & . & . \\
\hline Pterocarpus lucens & 835 & 8 & 823 & 8 & 795 & 12 & 780 & 15 & 794 & 34 & 730 & 3 & . & . \\
\hline Pterocarpus erinaceus & 688 & 17 & 624 & 15 & 618 & 6 & 603 & 27 & 621 & 24 & . & . & . &. \\
\hline Sclerocarya birrea & 519 & 8 & 509 & 7 & 497 & 8 & 490 & 12 & 467 & 21 & . & . & . & . \\
\hline Securinega virosa & 674 & 24 & 687 & 9 & & & & & . & . & . & . & . & 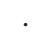 \\
\hline Sterculia setigera & 308 & 24 & 291 & 19 & 262 & 19 & 306 & 23 & . & . & . & . & . & . \\
\hline Strychnos spinosa & 711 & 15 & 669 & 11 & 639 & 12 & . & . & . & . & . & . & . & 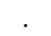 \\
\hline Tamarindus indica & 783 & 7 & 744 & 8 & 731 & 20 & . & . & . & . & . & . & 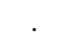 & - \\
\hline Terminalia avicennoides & 635 & 11 & 619 & 13 & & & . & . & . & . & . & . & . & . \\
\hline Ximenia americana & 668 & 9 & 641 & 9 & 664 & 20 & - & . & . & . & . & . & . & . \\
\hline
\end{tabular}

M: Mean.

SE: Standard error.

with increased tree height above $4.5 \mathrm{~m}$ the average $B D_{\mathrm{ub}}$ for discs per height increased for Anogeissus leiocarpus (table VI) which we believe was due to the need for structural stability in branches in the crown. Our results indicate that an increase in $B D_{\mathrm{ub}}$ occurred in the top of the stem for several other species i.e. Acacia seyal, Dalbergia melanoxylon, Prosopis africana and Pterocarpus erinaceus (table VI). 
The sampling system applied on each tree individual assumed an apical dominance with a clear main stem where discs values are given equal weights. For species with a bush stature bifurcating branches constitute a larger part of the total biomass than for species with a tree stature having a distinct main stem. Therefore increased weight for disc values up along the stem should be given depending on the amount of bifurcation for species with a bush stature. In this study no correction have been made for stem $B D_{\mathrm{ub}}$ for speciemens with more ramification. Less than a third of the 57 species in this study have a bush stature and six species in this study had a lianoid growth pattern with few major branches (table II).

Extraction of wood cores is a common procedure for determining the basic density. In this study wood cores would not be an option because of the small dimensions and, for many species, hard wood making extraction of good cores difficult. Moreover this would not provide an accurate assessment of the bark proportion because many savanna woody species having an irregular bark and wood surface. Therefore we believe that stem discs is an adequate sampling procedure for these conditions. Volume measurement under bark was made after debarking and this was difficult to make after the samples had dried whereas it was easy to debark freshly cut disc. Therefore volume determination was made in the forest on the site called Sa 14 .

In this study the time since the stands were cut was known and this is an advantage given the difficulty to determine age, by counting year rings, in tropical trees. However, within each stand, age was not homogeneous because stems continuously emerge and die. Therefore there is an age variation among sampled stems and we assume that the younger stems have smaller diameters. To examine the change in $B D_{\mathrm{ub}}$ and $B_{M \%}$ with $S D_{\mathrm{ub} 0.5}$ regression analyses were performed (table IV). For five species investigated there were indications of increased $B D_{\mathrm{ub}}$ and decreased $B_{M \%}$ with increased $S D_{\mathrm{ub} 0.5}$. Thus for these 5 species there were some evidence of juvenile wood and this has been reported in a previous study where density increased from pith to bark for 11 out of 18 dry Costa Rican forest species [17]. The order of magnitude of this change in bark and wood parameters can be exemplified with Anogeissus leiocarpus where the range of tree size $\left(D_{\mathrm{ub} 0.5}\right)$ in this study was about $100 \mathrm{~mm}(30-128)$. This corresponds to an increase of the $B D_{\text {ub }}$ of $17 \%$ (663 to 773) and a decrease of $B_{M \%}$ with $39 \%$ (28 to 17 ). However, this is clearly higher than what was estimated in a similar study in Ghana where bark proportion was only $7 \%$ in a 34 years old plantation of Anogeissus leiocarpus with a mean diameter at breast height of $9.8 \mathrm{~cm} \mathrm{[2].}$
In an analysis of the fuel-wood balance in Sahel, Jensen [10] used the same conversion factor for all species to obtain the oven-dry mass under bark from green woody volume over bark. Nevertheless, more accurate conversion factors can be obtained through weighting with the species-wise representation. Speciesspecific $B D_{\mathrm{ob}}$ information allows correcting for any bias due to the relative abundance of trees with different $B D_{\mathrm{ob}}$ and $B_{V \%}$ [8]. In this study a conversion figure has been calculated by weighting with the actual woody volume per species in the five stands (Nygård in prep.) and this resulted in a $B D_{\mathrm{ob}}$ of 0.68 ton $\mathrm{m}^{-3}(0.66-0.69)$ and a $B_{M \%}$ of $24 \%$ (20-25). Differences between sites representing different species composition appear to be small but when multiplied with the standing volume on a large scale the corrections can be considerable. Moreover we believe the $B D_{\mathrm{ob}}$ of 0.62 ton $\mathrm{m}^{-3}$ used by Jensen [10] is grossly underestimated considering it has been used also for old forests and data in this study indicates that $B D_{\mathrm{ob}}$ increase with dimension.

Data presented in this study could be used for discussions on ecological implications of rotation periods, silviculture and fuel-wood management. From a silviculture perspective, intensification of fuel-wood production should consider selective thinning of species with low $B D_{\mathrm{ub}}$ to improve the production of the remaining stand. There were indications within a given species that $B D_{\mathrm{ub}}$ increased and $B_{M \%}$ decreased with increased tree size $\left(D_{\text {ub } 0.5}\right)$. Hence, longer rotation periods would produce a better fuel-wood quality. Another reason for increasing the rotation period would be to reduce the bark proportion of the total biomass in order to reduce nutrient removal from the forest. According to Wang et al. [16] it is better to remove stem-wood > branches > bark to minimise nutrient removal from the forest. In this study $B_{M \%}$ of commonly used fuel-wood species in a young coppice forest constitute about a fourth of the total stem oven-dry mass and if bark is systematically harvested in large scale there is a risk of reduced long term site fertility. Could debarking of fuel-wood be a realistic silviculture option? According to Peltier et al. [13] a fuel-wood harvesting system is already in place in Niger to produce debarked fuel-wood, which is in fact demanded by the urban market [13]. By selecting the appropriate seasonal time of the year for cutting and storing the wood, debarking can be facilitated. Debarking could be considered a value adding processing of fuel-wood in rural areas where there is a lack of job opportunities. In this study the difference between $B D_{\mathrm{ob}}$ and $B D_{\mathrm{ub}}$ varied between species was indicating a higher bark basic density for some species (table III). Furthermore there were large variations between species in bark thickness and $M C_{\mathrm{ob} \%}$. High bark basic density, bark thickness and 
Table VII. Bark proportion, in percentage, on an ovendry matter basis, in percentage, per tree height in meter starting at stump for a savanna coppice forest in the age 5-14 years in Burkina Faso.

\begin{tabular}{|c|c|c|c|c|c|c|c|c|c|c|c|c|c|c|}
\hline \multirow[b]{3}{*}{ species } & \multicolumn{14}{|c|}{ tree height in meter } \\
\hline & \multicolumn{2}{|c|}{0,5} & \multicolumn{2}{|c|}{1,5} & \multicolumn{2}{|c|}{2,5} & \multicolumn{2}{|c|}{3,5} & \multicolumn{2}{|c|}{4,5} & \multicolumn{2}{|c|}{5,5} & \multicolumn{2}{|c|}{6,5} \\
\hline & $\mathrm{M}$ & SE & $\mathrm{M}$ & $\mathrm{SE}$ & M & SE & M & SE & M & SE & M & SE & $\mathrm{M}$ & SE \\
\hline Acacia ataxacantha & 14 & 1 & 18 & 1 & 22 & 1 & 24 & 1 & 22 & 1 & & & & \\
\hline Acacia dudgeoni & 32 & 1 & 34 & 1 & & & & & & & & & & \\
\hline Acacia gourmaensis & 30 & 1 & 31 & 2 & 24 & 7 & 9 & & 12 & & & & & \\
\hline Acacia macrostachya & 27 & 1 & 30 & 1 & 33 & 1 & 37 & 2 & 30 & & & & & \\
\hline Acacia pennata & 13 & 1 & 16 & 1 & 16 & & & & & & & & & \\
\hline Acacia senegal & 30 & 2 & 33 & 3 & 33 & 2 & & & & & & & & \\
\hline Acacia seyal & 23 & 1 & 25 & 1 & 26 & 1 & 27 & 1 & 30 & 2 & 31 & 3 & 35 & 2 \\
\hline Albizzia chevalieri & 25 & 3 & 27 & 3 & 38 & 5 & 46 & & & & & & & \\
\hline Anogeissus leiocarpus & 21 & 0 & 21 & 0 & 23 & 1 & 24 & 1 & 26 & 2 & 35 & 5 & & \\
\hline Balanites aegyptiaca & 33 & 2 & 32 & 2 & 30 & 2 & 22 & & 26 & & & & & \\
\hline Bombax costatum & 40 & 2 & 43 & 2 & 39 & 2 & 36 & 1 & & & & & & \\
\hline Boscia senegalensis & 31 & 1 & & & & & & & & & & & & \\
\hline Boswellia dalzielli & 35 & 2 & & & & & & & & & & & & \\
\hline Butyrospermum paradoxum & 30 & 1 & 32 & 1 & 35 & 2 & 36 & 2 & 36 & 0 & & & & \\
\hline Capparis sepiaria & 21 & 2 & & & & & & & & & & & & \\
\hline Cassia siberiana & 17 & 1 & 17 & 1 & 17 & 0 & 18 & & & & & & & \\
\hline Combretum fragrans & 24 & 2 & 26 & 1 & 24 & 2 & & & & & & & & \\
\hline Combretum glutinosum & 21 & 1 & 22 & 1 & 20 & 1 & 19 & 1 & & & & & & \\
\hline Combretum mircathum & 14 & 0 & 16 & 0 & 18 & 1 & 16 & 4 & & & & & & \\
\hline Combretum nigricans & 15 & 1 & 17 & 1 & 18 & 1 & 16 & 2 & & & & & & \\
\hline Commiphora africana & 35 & 1 & 32 & 1 & 32 & 1 & 39 & 3 & & & & & & \\
\hline Crossopteryx febrifuga & 25 & 2 & 29 & 2 & 29 & 3 & 31 & & & & & & & \\
\hline Dalbergia melanoxylon & 17 & 1 & 20 & 1 & 22 & 1 & 21 & 1 & 22 & 2 & & & & \\
\hline Detarium microcarpum & 42 & 5 & 47 & 4 & 46 & 4 & 44 & 1 & & & & & & \\
\hline Dicrostachys cinerea & 16 & 0 & 21 & 1 & 24 & 1 & 23 & & & & & & & \\
\hline Entada africana & 37 & 2 & 37 & 2 & 33 & 2 & 34 & 1 & 40 & & & & & \\
\hline Feretia apodanthera & 21 & 1 & 22 & 1 & 23 & 1 & 21 & & 24 & & & & & \\
\hline Grewia bicolor & 23 & 1 & 26 & 1 & 29 & 1 & 39 & 5 & & & & & & \\
\hline Grewia flavescens & 21 & 3 & 17 & 1 & 19 & & & & & & & & & \\
\hline Grewia mollis & 28 & 1 & 32 & 1 & 32 & 4 & 22 & & & & & & & \\
\hline Guiera senegalensis & 8 & 0 & 9 & 1 & 10 & 1 & 10 & & & & & & & \\
\hline Lannea acida & 53 & 2 & 54 & 1 & 53 & 2 & 54 & 5 & 49 & 13 & & & & \\
\hline Lannea mirocarpa & 39 & 1 & 41 & 1 & 39 & 1 & 40 & & & & & & & \\
\hline Piliostigma reticulatum & 28 & 2 & 29 & 2 & 34 & 2 & 36 & 3 & & & & & & \\
\hline Piliostigma thonningii & 35 & 2 & 35 & 2 & 35 & & & & & & & & & \\
\hline Prosopis africana & 24 & 2 & 26 & 1 & 27 & 1 & 25 & 1 & 28 & 2 & & & & \\
\hline Pterocarpus lucens & 14 & 1 & 17 & 1 & 18 & 1 & 20 & 1 & 14 & 10 & 24 & 1 & & \\
\hline Pterocarpus erinaceus & 34 & 3 & 37 & 3 & 27 & 1 & 30 & 2 & 28 & 1 & & & & \\
\hline Sclerocarya birrea & 28 & 1 & 31 & 1 & 33 & 1 & 34 & 2 & 37 & 1 & & & & \\
\hline Securinega virosa & 12 & 1 & 13 & 1 & & & & & & & & & & \\
\hline Sterculia setigera & 35 & 3 & 40 & 4 & 40 & 4 & 39 & 3 & & & & & & \\
\hline Strychnos spinosa & 19 & 2 & 22 & 1 & 24 & 0 & & & & & & & & \\
\hline Tamarindus indica & 22 & 1 & 25 & 2 & 29 & 5 & & & & & & & & \\
\hline Terminalia avicennoides & 38 & 3 & 39 & 2 & & & & & & & & & & \\
\hline Ximenia americana & 37 & 2 & 38 & 2 & 45 & 3 & & & & & & & & \\
\hline
\end{tabular}

M: Mean.

SE: Standard error.

$M C_{\mathrm{ob} \%}$ are essential for assessment of stem sensitivity to ground fire [14]. In Bolivia a bark thickness of $18 \mathrm{~mm}$ [14] at breast height was required to withstand lethal cambial temperatures in experimental low intensity fires.

\section{CONCLUSIONS}

There is a large variation in basic density between species in this study and the species composition varies 
strongly from one forest to another. Therefore conversion factors from standing woody volume to ovendry woody mass should be weighted with the species-wise representation. There were indications within a given species that basic density increased, and bark proportion decreased with increased tree size. This indicates that longer rotation periods will produce a woody biomass with higher basic density and lover bark proportion. Thus when evaluating fuel-wood production in a coppice forest, variations in species composition and tree age in a savanna forest must be considered.

Acknowledgements: We thank Centre National de la Recherche Scientifique et Technique and Ministère de l'Environnement in Burkina Faso for making this study possible and Y. Nouvellet at CIRAD-Forêt for cooperation with logistic and material. We are also grateful to Sören Holm for statistical advice. Funding was provided by Swedish International Development Cooperation Agency (Sida).

\section{REFERENCES}

[1] Abbot P.G., Lowore J.D., Characteristics and management potential of some indigenous firewood species in Malawi, For. Ecol. Manage. 119 (1999) 111-121.

[2] Adu-Anning C., Anglaaere L.C.N., Nwoboshi L.C., Growth, energy yield and nutrient uptake of some fuel-wood species in the Sudan savanna of Ghana, J. Tree Sci. 14, 1 (1995).

[3] Aubreville A., Flore forestière soudano-guinéenne, Office de la Recherche Scientifique Outre Mer. Société d'Éditions, Martimes et Coloniales, Paris (1950) p. 522.

[4] Bagnouls F., Gaussen H., Les climats biologiques et leur classification, Annales de Géographie 355 (1957) 193-220.

[5] Bellefontaine R., Gaston A., Petrucci Y., Aménagement des forêts naturelles des zones tropicales sèches, Cahier FAO Conservation 32 (1997).
[6] Borchert R., Soil and stem water storage determine phenology and distribution of tropical dry forest trees, Ecolog 75, 5 (1994) 1437-49.

[7] Catinot R., Aménager les savanes boisées africaines. Un tel objectif semble désormais à notre portée, Bois Forets Trop. 241 (1994) 53-69.

[8] Fearnside P.M., Wood density for estimating forest biomass in Brazilian Amazonia, For. Ecol. Manage. 90 (1997) 5987.

[9] Guinko S. Végétation de la Haute-volta. Thèse d'État, Université de Bordeaux III (1984).

[10] Jensen A.M., Évolution des données sur les ressources ligneuses au Burkina Faso, Gambie, Mali, Niger et Sénégal. In:Examen des politiques, stratégies et progra mmes du secteur des énergies traditionnelles, Banque Mondiale (Africa Technical Department), AFTPS (1995).

[11] Nouvellet Y., Évolution d'un taillis de formation naturelle soudano-sahélienne au Burkina Faso, Bois Forets Trop. 237 (1993) 45-79.

[12] Olesen P.O., The water displacement method a fast and accurate method of determining the green volume of wood samples, For. Tree Improv. 3 (1971).

[13] Peltier R., Betrand A., Lawali E.M., Madon G., Montagne P., marchés ruraux de bois-énergie au Sahel, Bois Forets Trop. 245 (1995) 75-83.

[14] Pinard M.A., Huffman J., Fire resistance and bark properties of trees in a seasonally dry forest in eastern Bolivia, J. Trop. Ecol. 13 (1997) 727-740.

[15] Tietema T., Ditlhogo M., Tibone C., Mathalaza N., Characteristics of eight firewood species of Botswana, Biomass and Bioenergy 1 (1991) 41-46.

[16] Wang D., Bormann H.F., Luego A.E., Bowden R.D., Comparison of nutrient use efficiency and biomass production in five tropical tree taxa, For. Ecol. Manage. 46 (1991) 1-21.

[17] Wieman M.C. Williamson G.B., Wood specific gravity gradients in tropical dry and montane rain forest trees, Am. J. Bot. 76, 6 (1989) 924-928.

[18] Zar J.H., Biostatistical analysis, 2nd edn, PrencticeHall Inc., Englewood Cliffs, N.Y., 1984. 